\title{
Study on the Fatigue Crack Growth Problem under Mode I+II Mixed Mode Condition with Elastic-Plastic Deformation*
}

\author{
Masanori KIKUCHI** and Toshiaki SATO**
}

\begin{abstract}
The fatigue crack growth test under mode II dominant condition is conducted. It is found that the crack growth direction can not be predicted by the conventional method. By the fracture surface observation, it is also found that the thickness effect strongly affects this behavior. Three dimensional elastic-plastic FEM analyses are conducted for this problem considering the Bauschinger effect. The change of the crack tip stress field during fatigue crack growth is studied. The criteria to predict the fatigue crack growth direction are studied and new criteria are discussed.
\end{abstract}

Key Words: Fatigue, Mixed Mode Loading, Crack Growth Path, Stress Ratio, FEM, Elastic-Plastic Analysis

\section{Introduction}

It has been widely known that the fatigue crack growth direction under mixed mode loading is well estimated by the Erdogan-Sih criterion ${ }^{(1)}$ where the crack growth is assumed to occur under the pure mode I condition $^{(2)-(6)}$. The crack growth direction is determined by the combination of mixed mode stress intensity factors, $K_{\mathrm{I}}$ and $K_{\text {II }}$. By using the equivalent stress intensity factor ${ }^{(7)}$, the crack growth rate is predicted using Paris' law ${ }^{(8)}$. Then the fatigue crack growth behavior under mixed mode condition is predicted well by the numerical simulation.

In the safety assessment of the nuclear power plant, the fatigue crack growth behavior under mixed mode loading should be evaluated. For this purpose, several conventional methods are proposed. Under small scale yielding condition, the fatigue crack under mixed mode loading condition changes the crack growth direction immediately after the initiation of the growth, and grows under pure mode I condition. In this paper, the fatigue crack growth with elastic-plastic deformation is studied. Tanaka et al. ${ }^{(9)}$ showed that the mixed mode fatigue crack growth occurs for aluminum alloy specimen under elastic-plastic deformation condition. The same phenomena was reported later by Otsuka et al. ${ }^{(10)}$ In these studies, effects of the mixed mode ratio, $K_{\mathrm{II}} / K_{\mathrm{I}}$, and the stress ratio of the cyclic load-

* Received 16th December, 2003 (No. 03-4201)

** Mechanical Engineering Department, Faculty of Science and Technology, Tokyo University of Science (RIKADAI), 2641 Yamazaki, Noda, Chiba 278-8510, Japan. E-mail: kik@me.noda.tus.ac.jp ing on the mixed mode fatigue crack growth direction are discussed experimentally. But the numerical studies on this problem have not been thoroughly done yet. In these experiments, plastic zone is nucleated around the crack tip. Then the stress and strain fields around the crack tip becomes very much complicated. By this reason, detailed numerical simulation has not been conducted for this problem, and the mechanical condition for the mixed mode crack growth is not yet clear.

In this paper, the experimental study is conducted at first. Then the fatigue crack surfaces are observed using Scanning Electron Microscope (SEM) precisely and the three dimensional numerical simulation is carried out. The condition of pure mode I crack growth and the mixed mode crack growth is discussed.

\section{Experiment}

\subsection{Testing condition}

In the experiment, slanted crack specimen is made as shown in Fig. 1. At first, a fatigue crack is introduced in a large plate by mode I cyclic loading. After then a test specimen is cut out from this large plate as shown in this figure, and the slant crack specimen is obtained. The loading axis in the mixed mode test is shown in this figure by arrows. The angle of the slant crack with respect to the loading axis is changed as $30^{\circ}, 35^{\circ}, 45^{\circ}$ and $60^{\circ}$. In the following, these specimens are called as $30^{\circ}$ specimen, $35^{\circ}$ specimen, and so on. $30^{\circ}$ is the critical angle for the mixed mode crack growth, which was found by Tanaka et al. ${ }^{(9)}$ In this paper, $30^{\circ}$ specimens are mainly tested and discussed. Test specimen material is aluminum alloy A2017, and the 


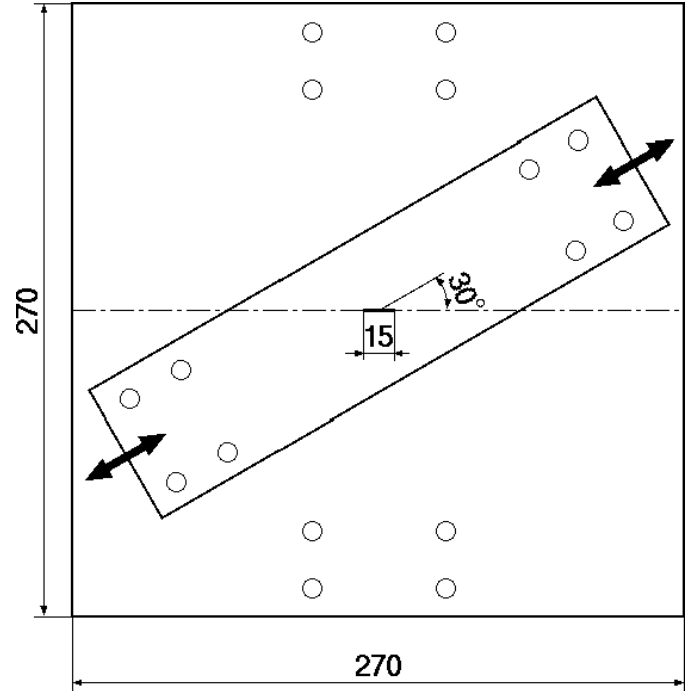

Fig. 1 Test specimen with a slant crack

Table 1 Mechanical properties of Aluminum alloy 2017

\begin{tabular}{|c|c|c|c|}
\hline $\mathrm{E}[\mathrm{MPa}]$ & $v$ & $\sigma_{\mathrm{Y}}[\mathrm{MPa}]$ & $\sigma_{\mathrm{B}}[\mathrm{MPa}]$ \\
\hline 70600 & 0.33 & 304 & 444 \\
\hline
\end{tabular}

thickness of the specimen is $3 \mathrm{~mm}$. The mechanical properties of the material are shown in Table 1.

The mixed mode ratio, $\Delta K_{\mathrm{II}} / \Delta K_{\mathrm{I}}$ is kept to be 1.69 for $30^{\circ}$ specimen where $K_{\text {II }}$ mode is dominant. It has been shown that mixed mode fatigue crack growth does not occur if this ratio is smaller than this value.

Three kinds of tests are conducted by changing the stress ratio $R$ as $R=0.1,0.3$ and 0.5 . The maximum stress is kept to be constant for all tests. $R=0.1$ means the stress range is large, and the stress intensity factor (SIF) range becomes large. Under this condition, elastic-plastic deformation occurs at the crack tip. $R=0.5$ is corresponding to fatigue test with small scale yielding condition. $R=0.3$ is medium between them. By Erdogan-Sih criterion, it is predicted that the pure mode I fatigue crack growth may occur along $-60^{\circ}$ direction with respect to the initial crack direction when the initial crack direction inclines $30^{\circ}$ with respect to the loading axis. For each $R$ value and angle, three specimens are tested. The fatigue crack growth process is observed using CCD camera, and the growing crack path and directions are measured.

\subsection{Experimental results}

Figure 2 shows the crack growth path of $R=$ 0.1 specimen, where $\Delta K_{\mathrm{I}}=212.0 \mathrm{MPam}^{1 / 2}$ and $\Delta K_{\mathrm{II}}=$ $359.4 \mathrm{MPam}^{1 / 2}$ are given respectively. From the pre-crack tip, the fatigue crack grows along $17^{\circ}$ direction with respect to the initial crack direction. This is not $-60^{\circ}$, and this is the mixed mode fatigue crack growth. It terminates after $3 \mathrm{~mm}$ growth. After then, another crack branches from the midpoint of the mixed mode fatigue crack path,

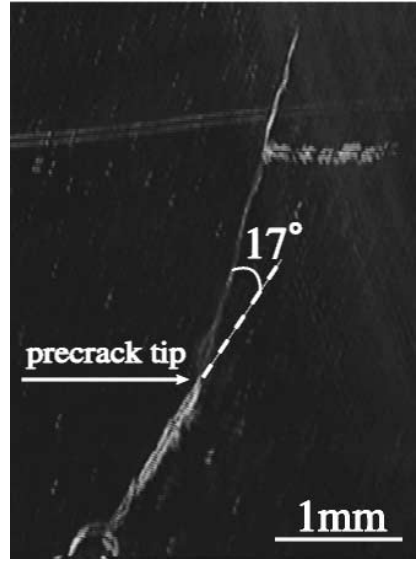

Fig. 2 Fatigue crack path of $R=0.1$ specimen

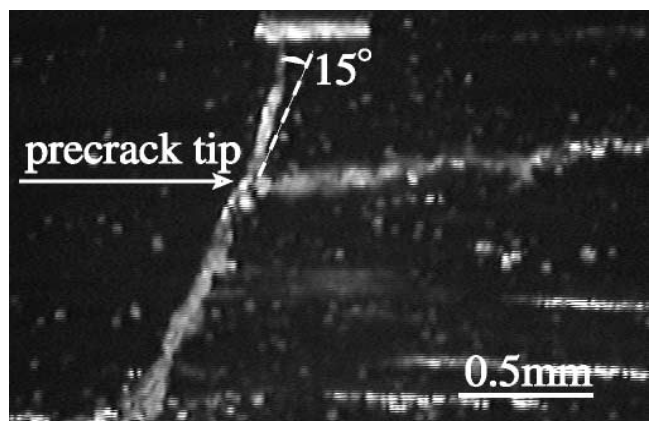

Fig. 3 Crack path of $R=0.3$ specimen

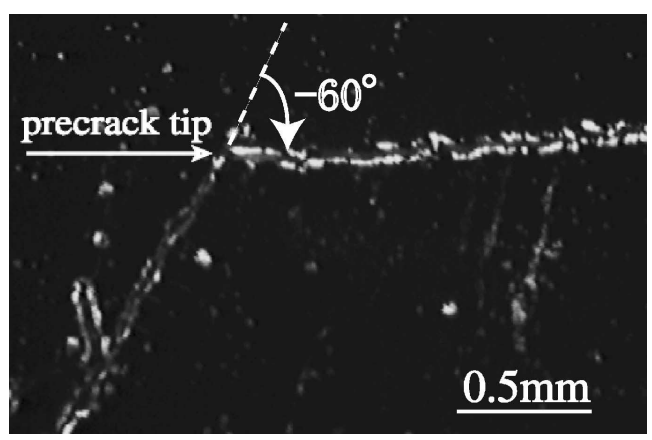

Fig. 4 Crack path of $R=0.5$ specimen

and grows toward $-60^{\circ}$ direction. This is the pure mode I fatigue crack. In Fig. $3, R=0.3\left(\Delta K_{\mathrm{I}}=164.9 \mathrm{MPam}^{1 / 2}\right.$ and $\Delta K_{\mathrm{II}}=279.5 \mathrm{MPam}^{1 / 2}$ ), the mixed mode fatigue also occurs. At first, the fatigue crack grows along $15^{\circ}$ direction and after $0.5 \mathrm{~mm}$ growth, it terminated. Then mode I fatigue crack is initiated from the pre-crack tip and grows toward $-60^{\circ}$ direction. In Fig. $4, R=0.5$ $\left(\Delta K_{\mathrm{I}}=117.8 \mathrm{MPam}^{1 / 2}, \Delta K_{\mathrm{II}}=199.7 \mathrm{MPam}^{1 / 2}\right)$, the fatigue crack does not grow along mixed mode direction. It grows along $-60^{\circ}$ direction from the initial pre-crack tip. Three specimens were tested for each $R$ values, and results are similar to each other. It is clear that for small $R$ value test, the mode I fatigue was not initiated at first. It grows under mixed mode condition. But after some amount of crack growth, the mode I fatigue crack growth occurs, and 


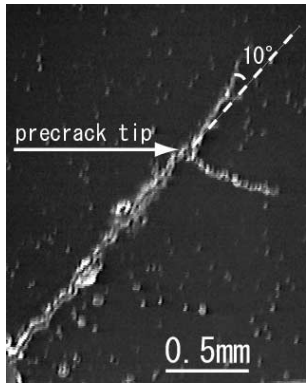

(a) $45^{\circ}$ specimen

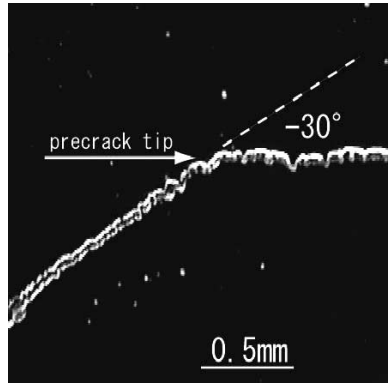

(b) $60^{\circ}$ specimen
Fig. 5 Crack paths of $R=0.1$ tests

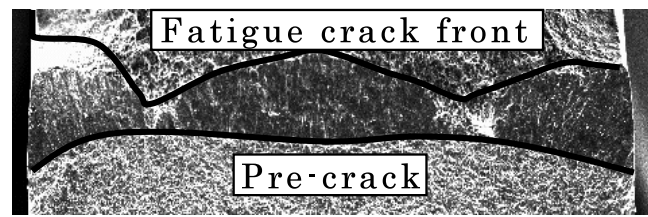

(a)

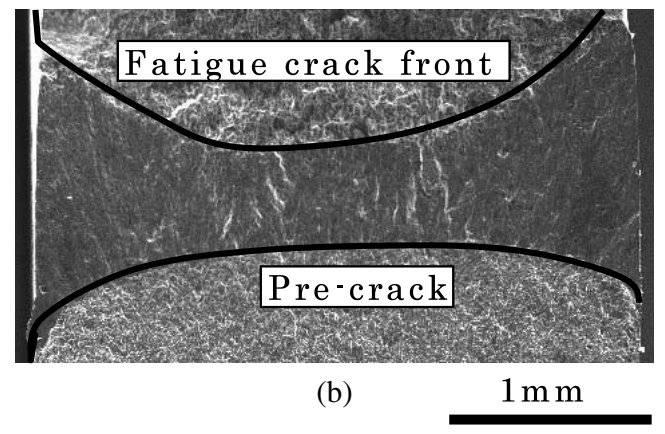

Fig. 6 Fatigue crack surfaces $\left(R=0.1,30^{\circ}\right)$

mixed mode growth terminates. Some mechanical condition changes during the mixed mode fatigue crack growth, and the condition of mode I growth becomes dominant.

Figure 5 (a) and (b) show the results of $45^{\circ}$ and $60^{\circ}$ specimens for $R=0.1$ loading conditions. In $45^{\circ}$ specimen, a small amount of crack growth along $10^{\circ}$ direction with respect to the initial crack direction is observed, and after then, the pure mode I fatigue crack is initiated and grows. But in $60^{\circ}$ specimen, the mixed mode crack growth does not occur at all. The pure mode I fatigue crack is initiated directly from the pre-crack tip.

Figure 6 (a) and (b) show the fatigue crack surface photos after the test. They are the results of $R=0.1$ tests of $30^{\circ}$ specimen. Figure 6 (a) is the photo of the specimen after small amount of fatigue crack growth. The cyclic load is stopped and the fatigue crack surface is observed. The fatigue crack growth is initiated from three locations on the initial crack front They are: at both specimen surfaces, and at the mid-plane of the specimen. From these locations, large amount of crack growth occurs at first, and they grow along the crack front, and coalesce with each other.

Figure $6(\mathrm{~b})$ is the photo after mixed mode fatigue

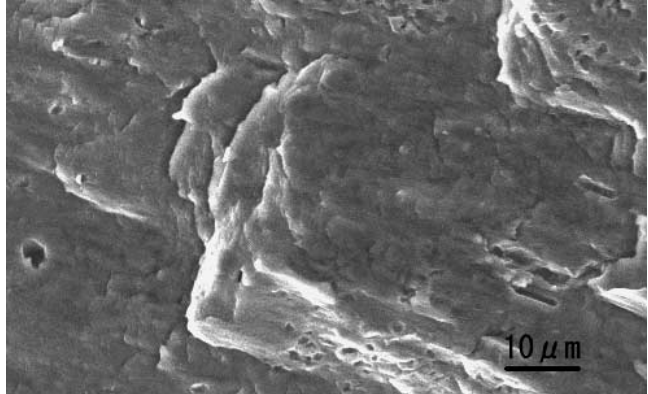

Fig. 7 Fatigue crack surface

crack growth terminated. This is also the result of $R=0.1$ of $30^{\circ}$ specimen. The mixed mode fatigue terminates after large amount of growth at both specimen surfaces, and crack growth amount is small at the middle of the specimen. The crack growth amount at the surface is more than 2 times larger than that at the mid-plane of the specimen. It may be concluded that the mixed mode fatigue crack growth is controlled mainly by the growth at the surface. It means that some mechanical parameter at the surface dominates these phenomena. In the general mode I fatigue crack growth, the tunneling effect is observed experimentally. But under mixed mode loading condition, the crack front configuration becomes largely different from that of mode I case. It is also shown that when the mode I fatigue crack growth is initiated, it starts at the middle of the specimen thickness and tunneling effect is also observed.

Figure 7 is the detailed photo of the fatigue crack surface by the mixed mode loading. Many thin laminae are observed on the fatigue crack surface. It is the characteristic feature of the mixed mode fatigue, which agrees with the experimental observation by Otsuka et al. ${ }^{(10)}$ There is no striation on this fracture surface. It is another evidence that this fatigue fracture is not controlled by the mode I loading.

\section{Numerical Simulation}

\subsection{Numerical model}

To study the change of the mechanical conditions around the crack tip, three dimensional FEM analyses are conducted. By the mode I pre-cracking, a curved crack front along the thickness direction is made by the tunneling effect. It is measured experimentally and is used in the finite element modeling. Figure 8 (a) and (b) show the models for the FEM analysis. This is the model of $30^{\circ}$ specimen. Half model is used due to the symmetry of the specimen configuration. In Fig. 8 (b), the curvature of the crack front along the specimen thickness is shown. Along the thickness direction, the specimen is divided in 5 layers.

The elastic-plastic stress-strain relation is approximated using following equation.

$$
\sigma=A\left(1+\varepsilon^{p}\right)^{n}
$$

where $\varepsilon^{p}$ is equivalent plastic strain and $A$ and $n$ are con- 


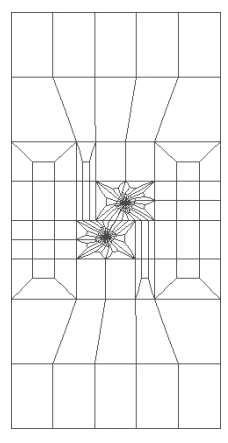

(a) Full model

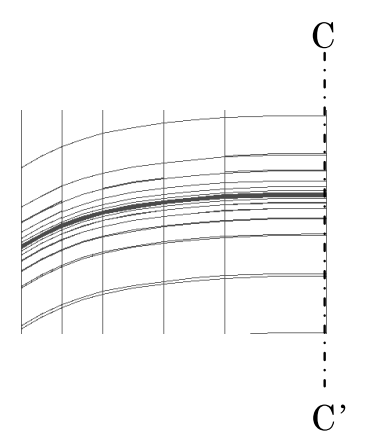

(b) Crack front curvature

Fig. 8 FEM model for MARC

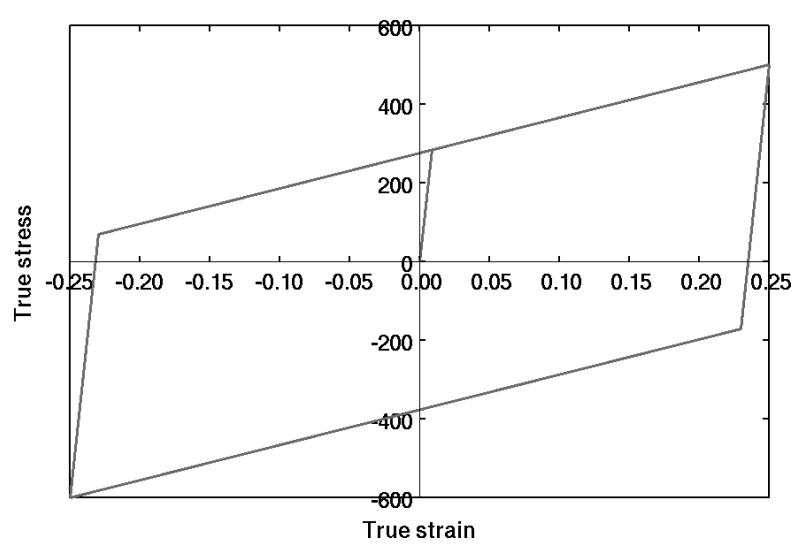

Fig. 9 Model of Bauschinger effect

stants. $A$ and $n$ vlaues are determined as $A=331.0 \mathrm{MPa}$ and $n=2.73$ by referring the experimental stress-strain curve. As the cyclic load is subjected in the elastic-plastic deformation state, the Bauschinger effect is taken into account for the numerical simulation. The Bauschinger effect is also measured experimentally, and is modeled as shown in Fig. 9. For finite element analyses, FEM package, MARC, is used.

Based on the experimental results, three problems on the mixed mode fatigue crack growth should be considered. They are: (a) the effect of stress ratio, $R$, (b) the effect of the angle of the crack with respect to the loading direction, and (c) the effect of the crack growth under mixed mode condition. By the numerical simulation, some mechanical parameters which correspond these phenomena are studied. In this paper, three parameters are mainly studied. They are maximum hoop stress, maximum shear stress and equivalent plastic strain. As the stress ratio has large effect on the experimental result, the absolute value of these parameters is not important. The important value is the range of them during one load cycle.

\subsection{Numerical results}

3.2.1 Effect of the stress ratio Figure 10 is the distributions of the range of hoop stress around the crack tip of $30^{\circ}$ specimen. The abscissa of this figure is the angle from the initial crack direction, where 0 degree is

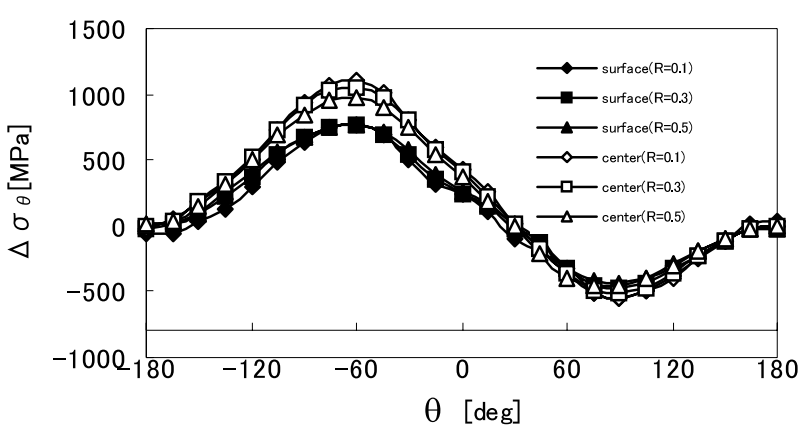

Fig. 10 Hoop stress range distribution of $30^{\circ}$ specimen

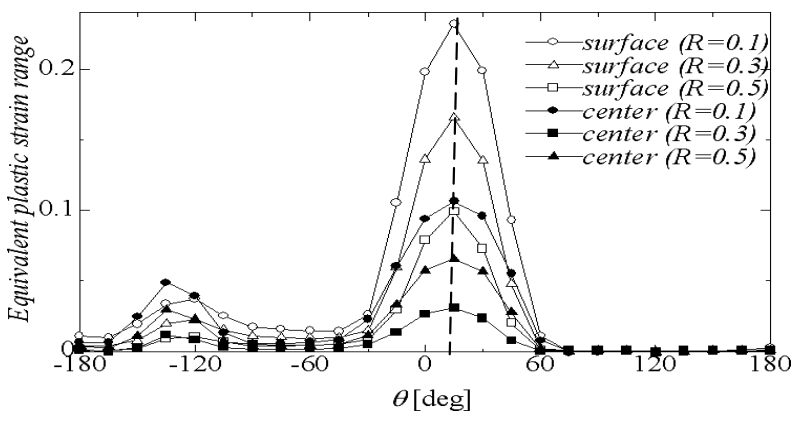

Fig. 11 Equivalent plastic strain range distribution for $30^{\circ}$ specimen

the initial crack direction. This is the result of the difference of the hoop stress at the maximum and the minimum stress states during one cycle loading. The results of three $R$ values and the values at surface and the midplane of the specimen are shown. For every condition, the peak value of the hoop stress range appears at $-60^{\circ}$ direction, which coincides with the direction predicted by Erdogan-Sih criterion. If the hoop stress determines the fatigue crack direction in the mixed mode condition, the crack may grow along this direction. But in the experiment, the mixed mode fatigue crack grows along $15-17^{\circ}$. It becomes obvious that the hoop stress is the dominant factor of the mode I fatigue crack growth, and is not dominant factor in the mixed mode fatigue crack growth. The mixed mode fatigue is controlled by other factors.

Figure 11 shows the ranges of the equivalent plastic strain at the crack front. Results of three $R$ values of $30^{\circ}$ specimens at mid-plane and surface are shown. For every case, the peak values appear at $18^{\circ}$ direction with respect to the initial crack direction. It agrees very well with the direction along which the mixed mode fatigue crack grows in the experiment. The peak value of $R=0.1$ specimen is much larger than those of $R=0.3$ and 0.5 specimens. It coincides with the experimental results that the mixed mode fatigue crack growth occurs in $R=0.1$ specimen, and does not grow in $R=0.5$ specimen. It is also noticed that the equivalent plastic strain at the surface is much larger than that of the mid-plane of the specimen. It also agrees with the experimental results that the mixed mode fatigue crack 


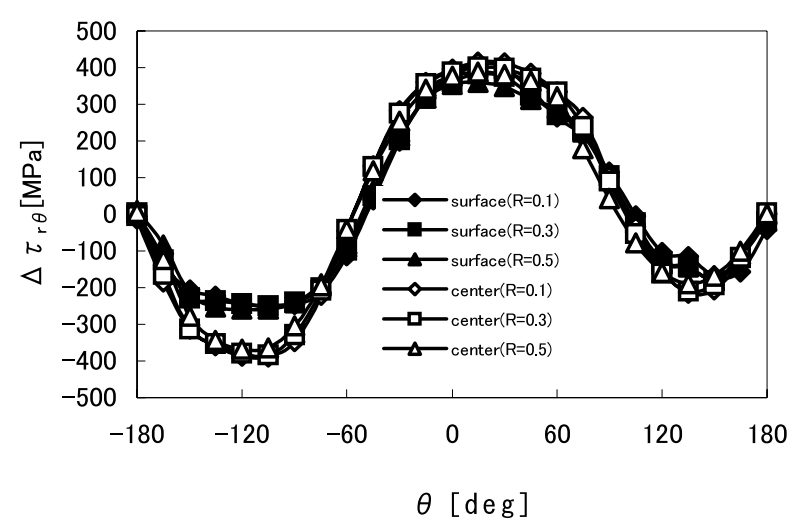

Fig. 12 Shear stress range distribution of $30^{\circ}$ specimen

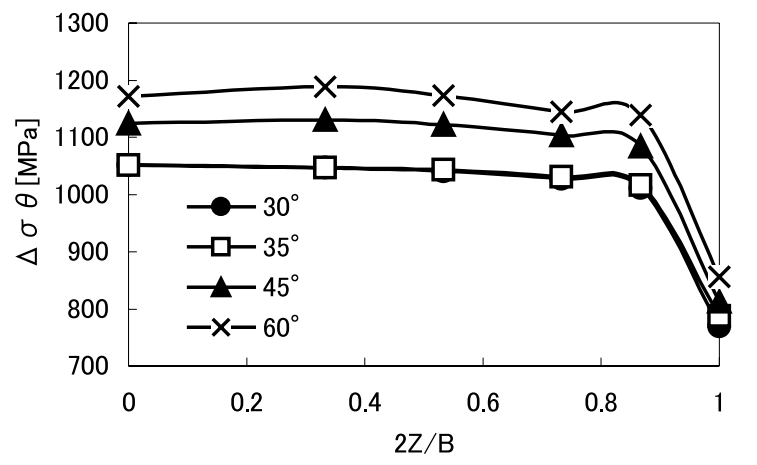

Fig. 13 Change of the hoop stress range with change of $\theta$ value

grows faster at the surface than at the mid-plane. It may be concluded that this parameter could be the dominant parameter for the mixed mode fatigue crack growth.

Figure 12 is the distributions of the range of the shear stress at the crack tip. This is also the results of $30^{\circ}$ specimen for three $R$ values. The peak values of them exist at about $18^{\circ}$ from the initial crack direction, which also agree with the experimental crack growth direction under mixed mode condition. But the effect of $R$ value is not clear in this figure. All results show similar curves, and the differences between the surface and the mid-plane are not clear. Then the range of the shear stress corresponds with the mixed mode fatigue crack direction, but it does not related with the $R$ effect and the fatigue crack growth at the surface of the specimen.

Comparing these results with experimental ones, it is estimated that the equivalent plastic strain is dominant at the beginning of the mixed mode fatigue crack growth, and the hoop stress becomes dominant during the crack growth, and the mode I fatigue crack growth begins.

3.2.2 Effect of the initial crack direction In the following, the angle between the initial crack direction and the loading axis is expressed by $\theta$. Figure 13 shows the changes of the distributions of the hoop stress range along the thickness direction with the change of the initial crack direction for $R=0.3$ condition. They are the maximum values around the crack tip. The abscissa of Fig. 13

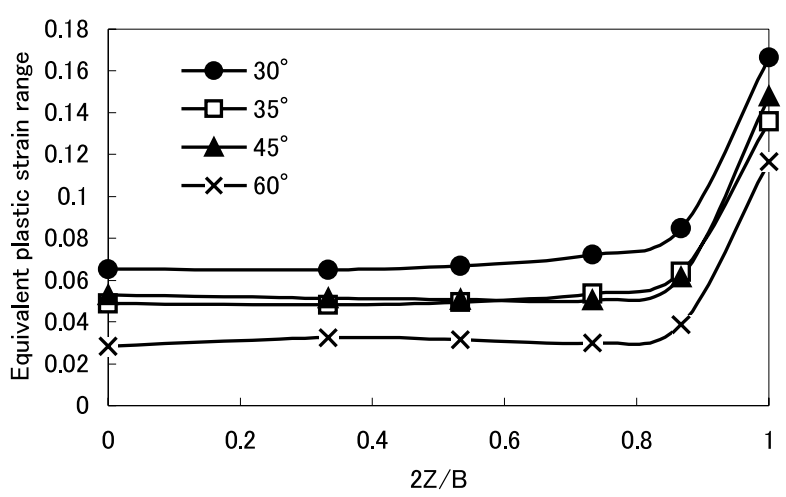

Fig. 14 Change of the equivalent plastic strain range with the change of $\theta$ value

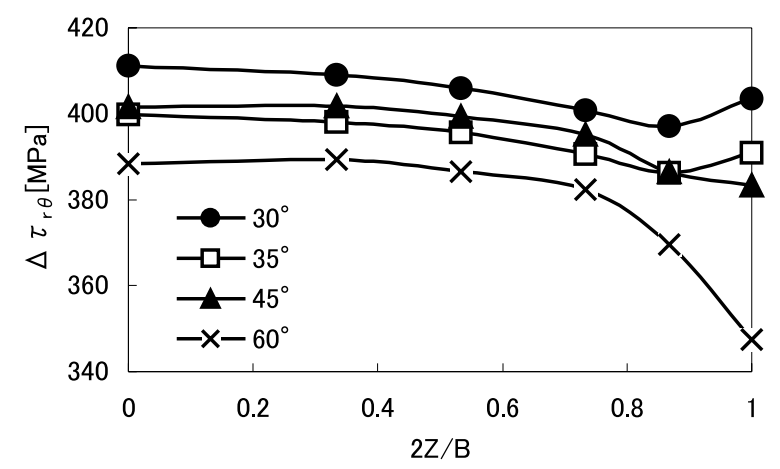

Fig. 15 Change of the shear stress range

means the location along the crack front normalized by the specimen thickness. $2 z / B=0$ means the mid-plane of the specimen and $2 z / B=1$ is the surface of it. In case of $30^{\circ}$ specimen, the hoop stress range is the smallest at every points along the specimen thickness. As the crack direction increases, the hoop stress range increases gradually, and becomes the maximum for $60^{\circ}$ specimen. These behaviors deeply related with the experimental result that the mode I fatigue crack growth occurs mainly for large $\theta$ specimens. It is also noticed that the hoop stress suddenly decreases near the surface of the specimen. It is estimated that the mode I crack growth occurs at the mid-plane at first. It agrees with many experimental observations.

Figure 14 is the changes of the equivalent plastic strain range with the change of $\theta$ value. They are the peak values around the crack tip. Contrary to the changes of the hoop stresses, the equivalent plastic strain range decreases with the increase of $\theta$ value. The maximum value along the specimen thickness is at the surface. This result means that the mixed mode crack growth may occur near the specimen surface for small $\theta$ value specimens, which agrees with the experimental results.

Figure 15 is the change of the shear stress range with the change of $\theta$ value. It also decreases with the increase of $\theta$ value, and nearly same for $35^{\circ}$ and $45^{\circ}$ specimens. This is the same tendency of Fig. 14. But shear stress range is not small inside of the specimen, which is a large differ- 
ence from the results of the equivalent plastic strain distribution.

Through these figures, it becomes obvious that due to the increase of $\theta$ value, hoop stress increases and the equivalent plastic strain and shear stress decrease. For small $\theta$ value specimen, the equivalent plastic strain or shear stress play the main roll for the mixed mode fatigue crack growth, but as $\theta$ value increases, these effects become small, and hoop stress plays the main role for the mode I fatigue crack growth.

\subsubsection{Effect of crack growth under mixed mode} condition The change of the dominant parameters during fatigue crack growth should be studied precisely by the numerical simulation. But the simulation of the fatigue crack growth with the cyclic elastic-plastic deformation is very much complicated. In this study, the simulation is conducted by a simplified manner. By changing the initial crack length, as shown in Fig. 16, four crack tip configurations are assumed, and one load cycle analysis is conducted for each model considering the elastic-plastic deformation. In the real specimen, the crack grows with the cyclic elastic-plastic deformation, and the stress and strain fields are the results of these stress-strain history. But in the following analyses, these history can not be taken into account. As shown in Fig. 16, it is assumed that the crack growth amount at the surface is $0.7 \mathrm{~mm}, 1.05 \mathrm{~mm}$ and $1.4 \mathrm{~mm}$, respectively. In the following, the assumed crack front configuration is called by this length. $0 \mathrm{~mm}$ means the original crack configuration. In the following, results for $R=0.3$ and $\theta=30^{\circ}$ specimens are shown.

Figure 17 is the distributions of the range of hoop stresses at the crack tip along the thickness direction for each crack configuration. The hoop stress decreases by the fatigue crack growth from the initial configuration to $0.7 \mathrm{~mm}$. After then it keeps nearly constant value by the further crack growth. The distribution pattern does not change largely by the fatigue crack growth. This is because the crack front configuration is self-similar for further crack growth. It is interesting to note that the hoop stress range does not increase largely by the further crack growth. At the mid-plane of the specimen, it takes the

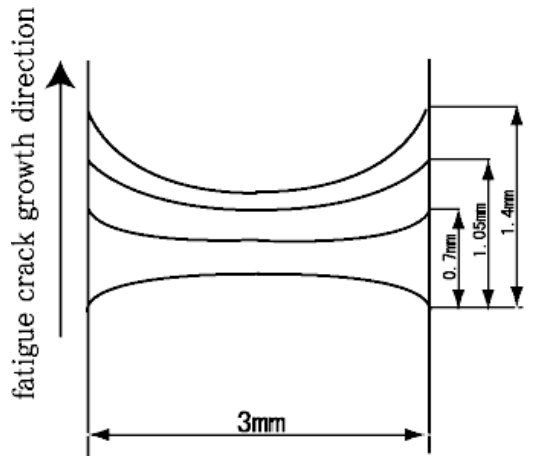

Fig. 16 Crack growth model maximum value.

Figure 18 shows the change of the equivalent plastic strain range distributions along the crack front by the crack growth. This value is the largest at the surface before crack growth. In Fig. 6(b), it is shown that the crack growth amount under mixed mode condition is the largest at the surface. It agrees with this numerical result. By the crack growth, it decreases largely only at the surface of the specimen. Finally, this value becomes the smallest at the surface of the specimen. The mode I fatigue crack is initiated from this final crack configuration. As estimated by the previous results, the equivalent plastic strain range has effect on the mixed mode fatigue crack growth behavior.

Figure 19 is the change of the shear stress range by the crack growth. Similar to Fig. 18, this value also decreases

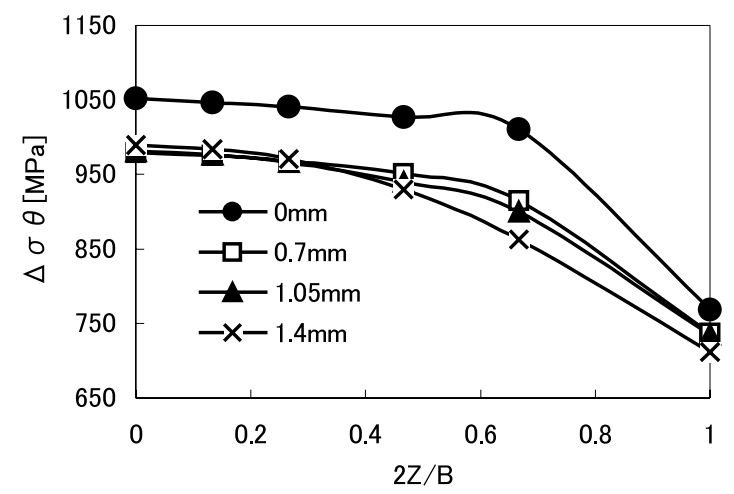

Fig. 17 Change of the hoop stress range by the crack growth

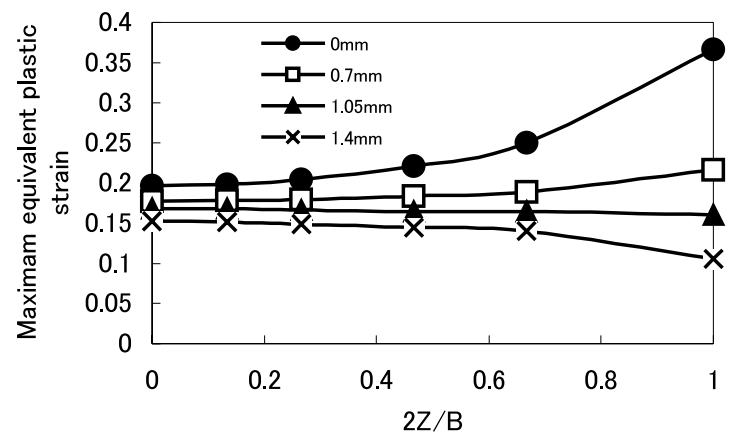

Fig. 18 Change of the equivalent plastic strain range by the crack growth

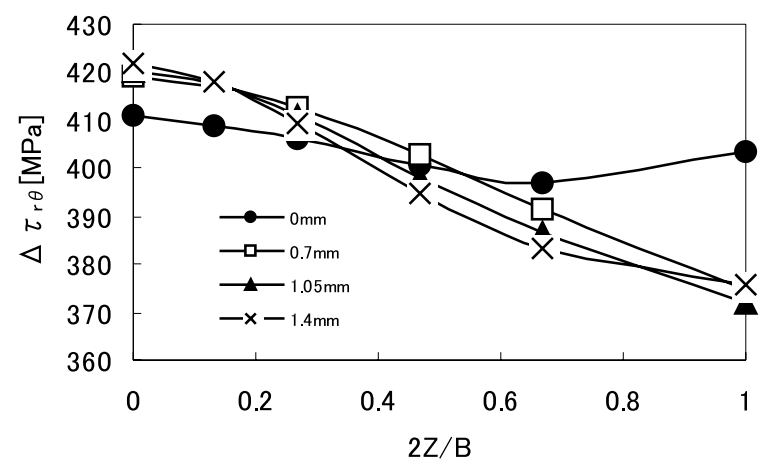

Fig. 19 Change of the shear stress range by the crack growth 
largely at the surface by the first step crack growth. At the mid-plane, it increases a little.

Through these results, the process of the fatigue crack growth is estimated as follows. At the beginning of the crack growth, the equivalent plastic strain at the surface of the specimen is dominant parameter, and the mixed mode fatigue crack growth occurs along the peak value direction of this parameter. But as the mixed mode fatigue crack grows, this parameter decreases rapidly at the surface. At the mid-plane, the hoop stress does not decrease and keeps nearly the same value. Then this parameter becomes dominant for the further crack growth, and mode I fatigue crack growth is initiated at the mid-plane.

The critical value of these two parameters are evaluated by further experimental study. Then the prediction of the transition of the crack growth mode from mixed mode to mode I may be possible by the numerical simulation.

\section{Concluding Remarks}

The mixed mode fatigue crack growth problem with elastic-plastic deformation is studied experimentally and numerically. It becomes obvious that the equivalent plastic strain range is the dominant parameter of the mixed mode fatigue crack growth, and hoop stress is the dominant for the mode I fatigue crack growth. Next step is to determine these criteria quantitatively. For this purpose, further studies are needed.

\section{Acknowledgement}

The present research was conducted as a cooperative work of MF committee in The Japan Welding Engineering Society supported by Tohoku Electric Power Co. Ltd., Tokyo Electric Power Co. Ltd., Chubu Electric Power Co. Ltd., Hokuriku Electric Power Co. Ltd., Shikoku Electric Power Co. Ltd., and Japan Atomic Power Co. Ltd.

\section{References}

(1) Erdogan, F. and Sih, G.C., On the Crack Extension in Plates under Plane Loading and Transverse Shear, J. Bas. Engng., Trans. ASME, Ser. D, Vol.85 (1963), pp.519-527.

(2) Kitagawa, H. and Yuuki, R., Stress Intensity Factors under Two-Dimensional Stress Field, Trans. JSME, (in Japanese), Ser. A, Vol.41 (1976), pp.1641-1647.

( 3 ) Palaniswamy, K. and Knauss, W.G., On the Problem of Crack Extension in Brittle Solids under General Loading, Mechanics Today, Vol.4, (1978), p.30, Pergamon.

(4) Patel, A.B. and Pandey, R.K., On the Fatigue Crack Growth Problem under Mixed Mode Loading Condition, Fatigue Engng. Mater. Structures, Vol.4 (1981), pp.65-77.

( 5 ) Yong, J., Yates, J.R. and Brown, M.W., The Formation and Propagation of Mode I Branch Cracks in Mixed Mode Fatigue Failure, Engng. Fracture Mech., Vol.56 (1997), pp.213-231.

(6) Kikuchi, M., Transactions, Fatigue Test under Mode II+III Mixed-Mode Condition, SMiRT 16, Vol.G, (2001), \#1126.

( 7 ) Oji, K., Tuji, M., Kubo, S., Ono, Y., Yawata, A. and Umei, K., Predictions of Fatigue Crack Propagation Path and Life of High-Tension Steel in Residual Stress Fields, Trans. JSME, Ser. A, Vol.59 (1993), pp.14291434.

( 8 ) Paris, P.C. and Erdogan, F., A Critical Analysis of Crack Propagation Laws, J. Bas. Engng. Trans. ASME, Ser. D, Vol.85 (1963), pp.528-533.

(9) Tanaka, K., Fatigue Crack Propagation from a Crack Inclined to the Cyclic Tensile Axis, Engng. Fracture Mech., Vol.6 (1974), pp.493-507.

(10) Otsuka, A., Mori, K. and Miyata, T., The Condition of Fatigue Crack Growth in Mixed Mode Condition, Engng. Fracture Mech., Vol.7 (1975), pp.429-439. 